\title{
Diagnostic Sensitivity of Bronchoalveolar Lavage versus Lung Fine Needle Aspirate
}

\author{
Bradly D. Clark, M.D., Phyllis R. Vezza, M.D., Christie Copeland, C.T., Anna-Marie Wilder, C.T., \\ Andrea Abati, M.D. \\ Cytopathology Service, Laboratory of Pathology, National Cancer Institute/National Institutes of Health \\ (BDC, CC, A-MW, AA), Bethesda, Maryland; and Department of Pathology, Our Lady of Fatima Hospital \\ (PRV), North Providence, Rhode Island
}

Bronchoalveolar lavage (BAL) and lung fine-needle aspirate (LFNA) are commonly performed as the first line of investigation for a myriad of pulmonary problems associated with abnormal imaging findings (mass, cavitary lesion, infiltrates, etc.). The relative sensitivities of these two procedures are not well established for cytologic diagnosis of lesions for any single disease event. Records were searched for single pulmonary disease events with closely timed BAL and LFNA, as defined by both procedures occurring within $\leq \mathbf{8}$ days of each other. No samples with "unsatisfactory" diagnoses were considered for the analyses. Success of identifying malignancy and/or an infectious agent was recorded for both procedures. Between January 1989 and June 2000, 52 episodes of closely timed (65\% within $3 \mathrm{~d}$ ) BAL and LFNA procedures were identified in 45 patients for a single disease event. The clinical scenarios as per the sample requisitions were as follows: consolidation/infiltrate $(60 \%)$, mass/nodule $(23 \%)$, cavitary lesion $(5.7 \%)$, pneumonia $(5.7 \%)$, or not specified $(5.7 \%)$. For all cases examined $(n=$ 52), in 18 (35\%) of the episodes, LFNA uniquely identified either malignancy, 6/18 (12\%), or infectious agents such as Aspergillus and acid-fast bacteria, 12/18 (23\%), with a corresponding nondiagnostic BAL. In one episode with a clinical diagnosis of infiltrates, the BAL was positive for acid-fast bacteria, whereas the LFNA was negative. Chi-square analysis of the data revealed statistical significance with $P<.0001$ with 2 degrees of freedom, indicating LFNA to be a superior method for the diagnosis of pulmonary pathology over BAL. Based on our data, LFNA is the superior method for the cytologic diagnosis of pulmonary pathology amenable to cytologic examination.

Copyright () 2002 by The United States and Canadian Academy of Pathology, Inc.

VOL. 15, NO. 12, P. 1259, 2002 Printed in the U.S.A.

Date of acceptance: July 10, 2002.

Address reprint requests to: Andrea Abati, M.D., Cytopathology Section, Lab of Pathology/NCI/NIH, Building 10, Room 2A19, Bethesda, MD 20892-1500; e-mail: abatia@mail.nih.gov; fax: (301) 402-2585.

DOI: 10.1097/01.MP.0000036456.45865.A0
KEY WORDS: Bronchoalveolar lavage, Immunosuppression, Lung fine needle aspiration, Respiratory cytopathology.

Mod Pathol 2002;15(12):1259-1265

Bronchoalveolar lavage (BAL) and lung fine-needle aspirate (LFNA) are commonly performed as the first line of investigation for a myriad of pulmonary problems associated with abnormal imaging findings (mass, cavitary lesion, infiltrates, etc.). BAL entails advancing a fiberoptic bronchoscope distally into a subsegment bronchus, followed by instillation of saline, which is retrieved along with cellular material from the lung (1). The complications arising directly from BAL are generally minor and may include benign postbronchoscopy fever, short-lived declines in $\mathrm{Pa}_{\mathrm{O} 2}$, laryngospasm, and, rarely, pneumothorax, hemothorax, or exacerbations of respiratory failure $(1,2)$.

LFNA involves percutaneous introduction of a fine needle $(<20$ guage) into the lung, with visual assistance provided by computed tomography (CT; 3 ). The mortality of LFNA is $0.1 \%$, as quoted in a combined series of 1562 patients (4). Other investigators have had no deaths occur in their experience of $>3000$ thoracic FNA procedures (5). Although fatalities may be rare, the rate of pneumothoraces secondary to LFNA reported in the literature varies in the range of 6 to $57 \%$ (6). Hemoptysis or hemorrhagic complications range from 3 to $10 \%$ (7). Other complications, such as air embolism and hemothorax, are extremely rare after LFNA (5).

Previous studies have demonstrated high positive predictive values of LFNA for the diagnosis of lung cancer (8). The true-positive rates for primary lung malignancies using LFNA (95 to 97\%) and bronchial washings (60 to $61 \%$ ) have remained consistent over the past 15 years (9), evidencing the superiority of LFNA as a diagnostic modality for the demonstration of malignancy. Interestingly, several studies in the 1980s demonstrated that BAL of immunosuppressed patients resulted in excellent 
sampling with high diagnostic yield and greater ease of differentiating viral and bacterial infections (10-13). However, the relative cytologic diagnostic sensitivities of these two procedures performed on the same patient for any single disease event (infectious or neoplastic) is not well established.

Past studies at the National Institutes of Health (NIH) have demonstrated experience with cytologic examination and diagnosis of pulmonary specimens from patients with chronic granulomatous disease (CGD), a rare inherited disorder in the pathway involving the oxidation of NADPH for the production of superoxide in phagocytic cells (14). Specimens from such patients could be prepared and completed, including fungal stains, within several hours from the time of specimen acquisition (15). Such expediency allows for prompt treatment of suspected fungal infections in the immunocompromised CGD patients. Cytology has proven to be highly sensitive in identifying fungus in patients with CGD. In fact, cytology has identified these infections in CGD patients with twice the success rate as have fungal cultures in the microbiology lab (16).

Studies of cytologic specimens from patients with CGD led us to identify patient cases in which concurrent BAL and LFNA were performed for the same disease episode. In turn, this prompted us to retrospectively review all patient cases, regardless of the patient's preexisting condition, for closely timed BAL and LFNA diagnostic studies. The aim of this study was to compare the results of BAL and LFNA performed at close intervals on the same patient for a single disease event in an attempt to determine whether one technique was superior to the other.

\section{MATERIALS AND METHODS}

Cytology records from January 1989 to June 2000 at the NIH Clinical Center were searched for single pulmonary disease events with closely timed BAL and LFNA, as defined by both procedures occurring within $\leq 8$ days of each other. The patient population of the NIH Clinical Center is protocol driven, with a majority of the patients having known malignancies, a primary immunosuppressive disease, or treatment-induced immunosuppression. Patient episodes were included in the study only if the clinical differential diagnoses of the BAL and the LFNA were similar for the closely timed procedures (i.e. $<8 \mathrm{~d}$ ). No samples with "unsatisfactory" diagnoses were considered for the analyses. Other than the timing of $\leq 8$ days between procedures, no other selection criteria were used to determine which episodes were chosen for this analysis.

Because this study is retrospective, covering a 10 -year period, not all of the BAL and LFNA speci- mens were treated with exacting uniformity. Currently, when the NIH cytopathology lab receives a BAL specimen, it is first triaged by a cytotechnologist by reading the accompanying history and evaluating two Diff-Quik cytospins. Extra cytospin preparations may be prepared for special studies, as determined by the cytopathologist. Typically, six more cytospins are made to use for Pap, silver, and acid-fast stains. At the NIH, the cytotechnologist assists the clinician in the collecting and fixation of all LFNA specimens. On returning to the laboratory, the cytotechnologist will have several different parts of the specimen to process: air-dried smears, alcohol-fixed smears, needle rinsings, and components of needle aspirates suspended in saline. If the sediment volume of the rinsing is scant, then only two cytospins are prepared. Should the rinse hold a large volume, it is used to prepare a cell block. Both air-dried smears and cell block paraffin-based sections are used for silver and acid-fast stains. The bronchoscopist or interventional radiologist makes the determination as to which specimen is forwarded to the microbiology lab.

We recorded diagnoses from both of the diagnostic procedures to track the success of identifying malignancy and/or an infectious etiologic agent. Other data, such as clinical diagnosis, clinical history, and dates of the procedures, was also acquired from the cytopathology reports.

\section{RESULTS}

Fifty-two episodes of closely timed BAL and LFNA procedures were identified in 45 patients for any single disease event. The interval between the procedures ranged from 0 to 8 days $(65 \%$ within 3 days; Table 1). In a majority of the episodes, 30/52 (58\%), the BAL preceded the LFNA. The BAL was performed the same day as the LFNA in 7 of the cases $(13 \%)$, and the BAL was performed after the LFNA in 15 of the 52 episodes (29\%).

Most of the patients, 25/45 (56\%), had an immunosuppressive condition. The remaining patients

\begin{tabular}{|c|c|c|c|}
\hline $\begin{array}{c}\text { Days } \\
\text { Elapsed } \\
\text { between } \\
\text { Procedures }\end{array}$ & $\begin{array}{c}\text { Patient } \\
\text { Episode } \\
\text { Count }\end{array}$ & $\begin{array}{c}\text { Cumulative } \\
\%\end{array}$ & $\begin{array}{c}\text { Individual } \\
\%\end{array}$ \\
\hline 0 & 7 & 13.5 & 13.5 \\
\hline 1 & 13 & 38.5 & 25.0 \\
\hline 2 & 9 & 55.8 & 17.3 \\
\hline 3 & 5 & 65.4 & 9.6 \\
\hline 4 & 6 & 76.9 & 11.5 \\
\hline 5 & 5 & 86.5 & 9.6 \\
\hline 6 & 3 & 92.3 & 5.8 \\
\hline 7 & 1 & 94.2 & 1.9 \\
\hline 8 & 3 & 100.0 & 5.8 \\
\hline
\end{tabular}

BAL, brochoalveolar lavage; LFNA, lung fine needle aspiration. 
had primary malignancies, 14/45 (31\%); had a combination of immunosuppressive condition plus malignancy $2 / 45$ (4\%); or did not have a diagnostic category specified, $4 / 45$ (9\%).

The volume of lavage fluid arising from the BAL procedures transported to our cytopathology lab was reported in 48 of the 52 cases. The fluid volume ranged from $3 \mathrm{~mL}$ to $50 \mathrm{~mL}$, whereas the median volume reported was $12 \mathrm{~mL}$. Most of the BAL specimens were processed by cytocentrifugation (50 of 52). The number of slides resulting varied from 2 to 10 slides, with 4 slides being the median number made from cytospins. Other kinds of preparations were made, such as Millipore filtrations, liquidbased slides, and cell blocks. However, these other methods were used with variation and were not consistently prepared for every case during the years 1989 to 2000 .

The clinical scenarios for the diagnostic episodes were as follows: consolidation/infiltrate (60\%), mass/nodule (23\%), cavitary lesion $(5.7 \%)$, pneumonia (5.7\%), and not specified (5.7\%; Fig. 1). For all cases examined $(n=52)$, in $18(35 \%)$ of the episodes, LFNA uniquely identified either malignancy, 6/18 (12\%), or infectious agents, 12/18 (23\%), with a corresponding non-diagnostic BAL (examples shown in Figs. 2-5). Of the six malignancies uniquely identified by LFNA, two of them were
non-Hodgkin's lymphomas, and the remainder were single diagnoses each of non-small cell carcinoma, Kaposi's sarcoma, squamous cell carcinoma, and metastatic renal cell carcinoma. Fungal elements, usually Aspergillus species, were discovered in 11 of the 12 cases in which LFNA identified infectious disease. The remaining case was positive for acid-fast bacteria. Six of the 12 episodes positive for infection involved patients with CGD. Because all data were not available, comprehensive comparison of results of cultures from microbiology to the BAL/LFNA discoveries was not made.

A closer look at the data demonstrates that 7 of 12 (58\%) infectious agents and three of the six (50\%) neoplasias were identified only by LFNA on episodes of BAL and LFNA with $\leq 3$ days elapsing between the two procedures. Five of six of the patients with chronic granulomatous disease were among those identified as positive for fungus only by LFNA with $\leq 3$ days elapsed between BAL and LFNA.

Of the seven episodes in which the BAL and LFNA were performed the same day, a single infectious agent (Aspergillus) and a single neoplasm (metastatic renal cell carcinoma) were identified in two separate patients by LFNA, whereas the BAL was nondiagnostic. Furthermore, in 15 of the procedures yielding diagnoses, the LFNA preceded the BAL. In one of those episodes, the BAL procedure

\section{Count of episodes by clinical findings}
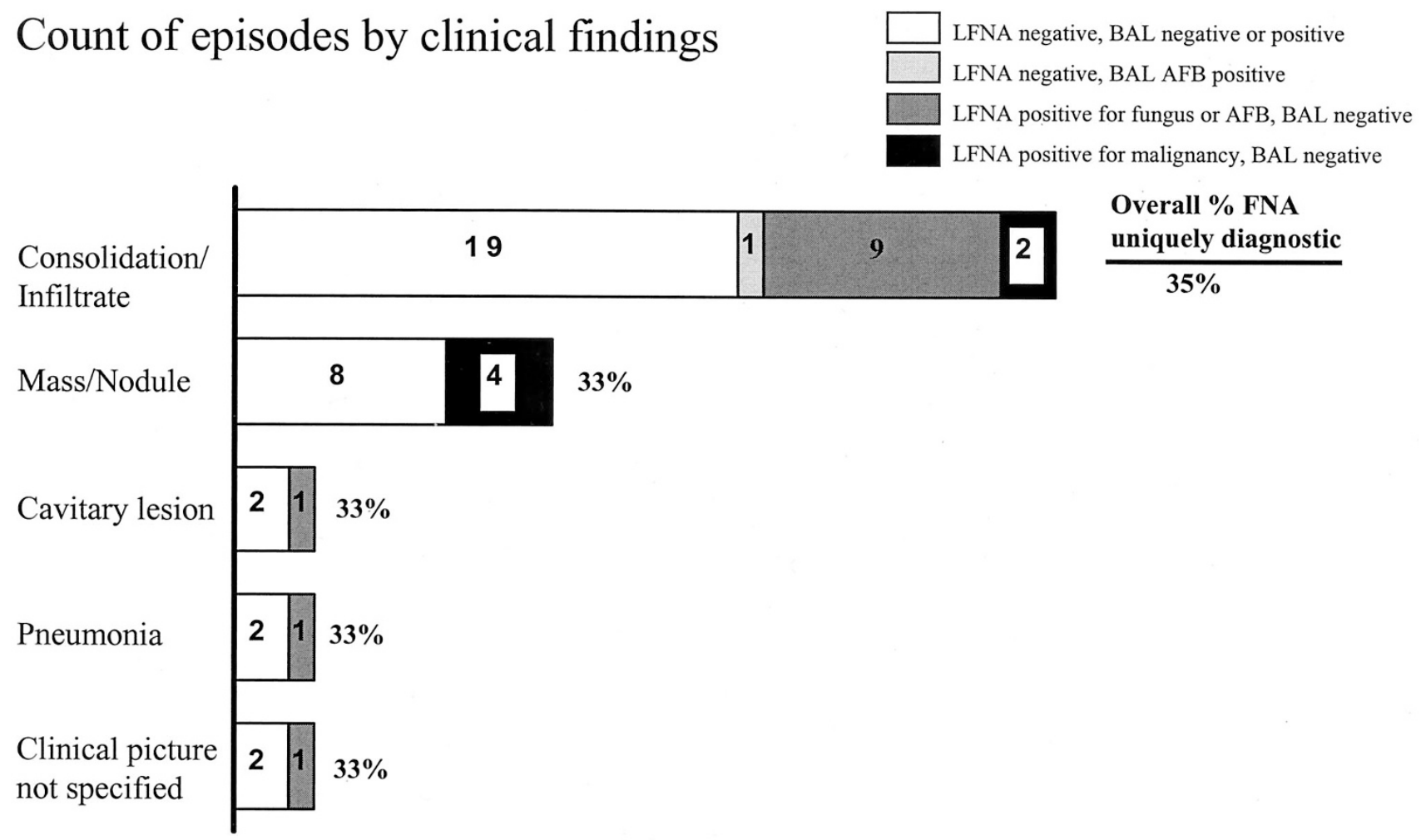

\begin{tabular}{l}
$\begin{array}{l}\text { Overall \% FNA } \\
\text { uniquely diagnostic }\end{array}$ \\
\hline $35 \%$
\end{tabular}

* Interval of days between BAL and FNA per patient per clinical diagnosis not exceeding 8 days.

BAL - Bronchoalveolar lavage; LFNA - Lung fine needle aspiration

FIGURE 1. Episodes of closely timed BAL and LFNA procedures, January 1989 to June 2000. 


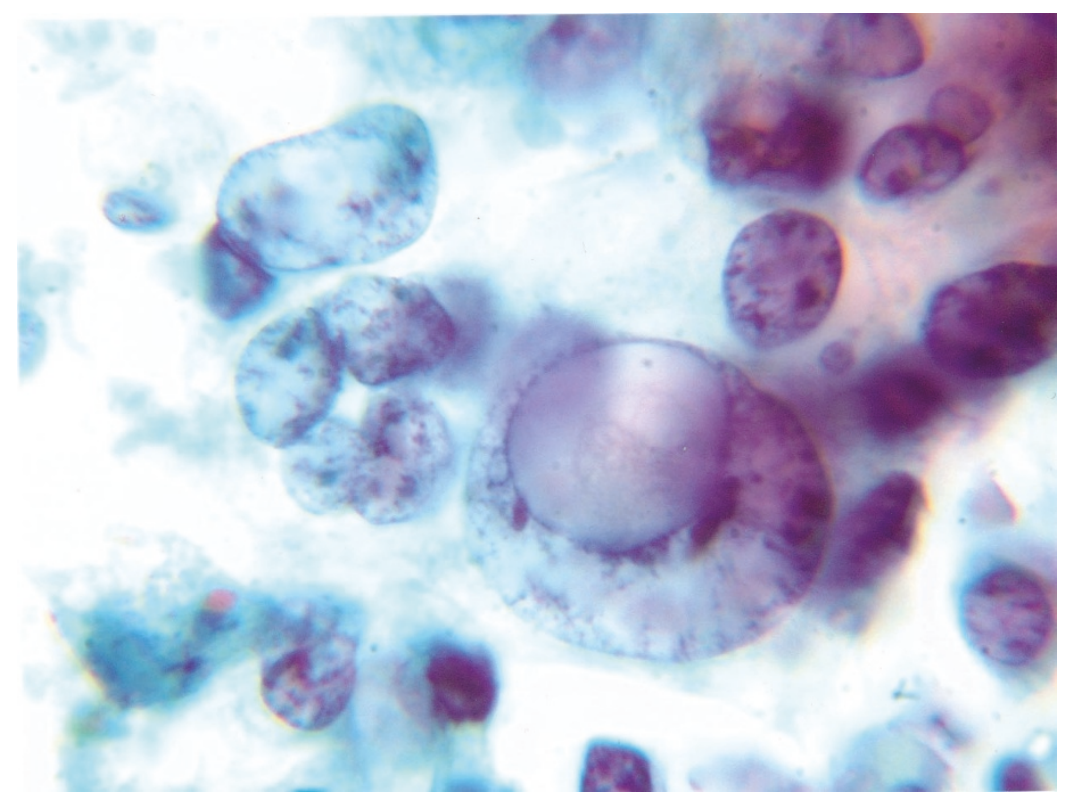

FIGURE 2. LFNA: pleomorphic cells with cytoplasmic intranuclear inclusions, round nuclei, and delicate cytoplasm. Adenocarcinoma (1000×, Papanicolaou staining).

was positive for acid-fast bacteria, whereas the FNA was negative. In $3 / 15$ of those episodes, LFNA uniquely identified fungal forms on three separate

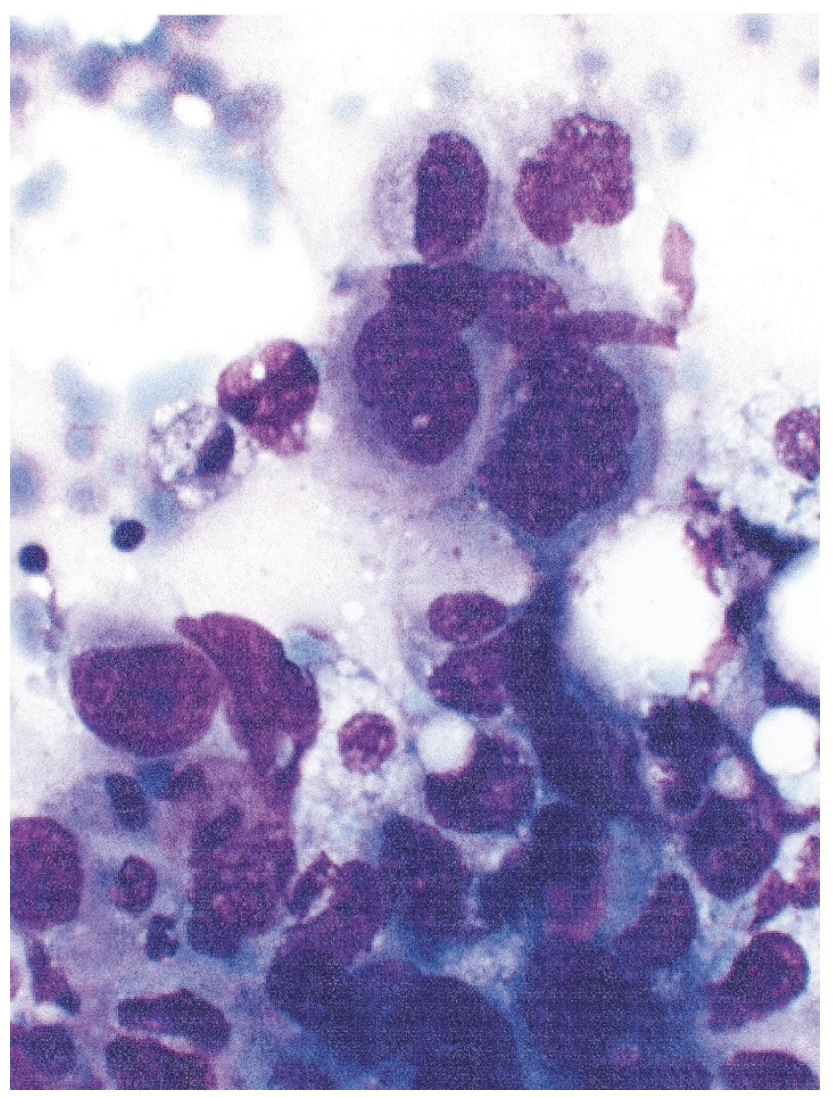

FIGURE 3. LFNA: sheets of atypical cells with irregular nuclear contours, nuclear hyperchromasia, basophilic cytoplasm, and jagged outline of cell borders. Squamous cell carcinoma (400×, Diff-Quik staining). patients. LFNA also uniquely demonstrated squamous cell carcinoma in yet another patient from those 15 episodes in which LFNA took place before BAL.

When the patient episodes were segmented by clinical findings, LFNA uniquely identified either malignancy or infection in one third of the cases demonstrating a consolidation/infiltrate, a mass/ nodule, pneumonia, or a cavitary lesion (not statistically significant; Fig. 1). As mentioned previously, in one episode with a clinical diagnosis of infiltrates, the BAL was positive for acid-fast bacteria, whereas the LFNA was negative for both infection and malignancy. There were no cases found in which a patient was diagnosed by cytology with simultaneous malignancy and infection.

Chi-square test for independence of the data (performed using Microsoft Excel; Microsoft, Redmond, WA) revealed statistical significance with $P<.0001$ with 2 degrees of freedom, indicating LFNA to be a superior method for the diagnosis of pulmonary pathology over BAL in our data set (Table 2).

During this study, we were limited to a finite set of data. This prevented access to the complete medical records of the patients evaluated. As a result, we were not able to compare the relative costs of the procedures per patient or discover any untoward effects that the patients may have experienced resulting directly from being evaluated by BAL and/or LFNA.

\section{DISCUSSION}

The patient population of the NIH Clinical Center is protocol driven, with the majority having known 


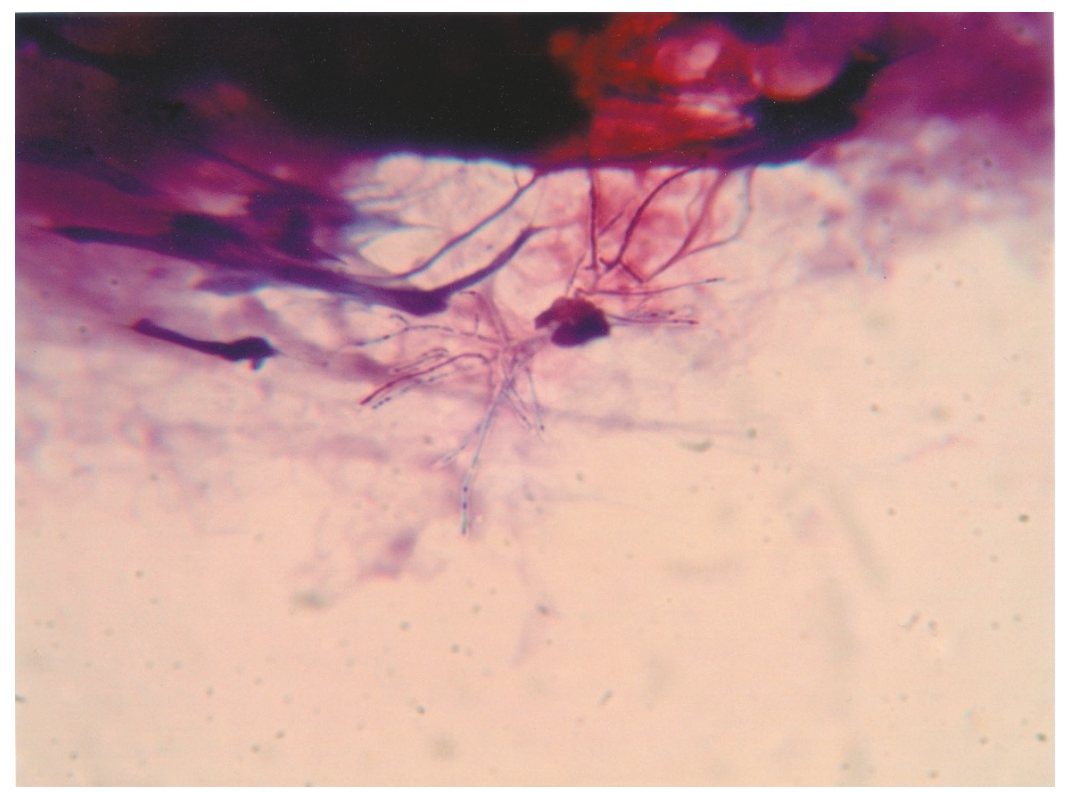

FIGURE 4. LFNA: modified Fite stain showing numerous filamentous beaded bacteria. Nocardia (1000×, modified Fite staining).

malignancies, a primary immunosuppressive disease, or treatment-induced immunosuppression. In a comparison of patients with pulmonary pathology limited to neoplasia or infection, LFNA was the superior diagnostic modality for determination of the pulmonary disease process.

In patients in whom malignancy or opportunistic infection is suspected in the context of diffuse parenchymal disease, the current recommendations suggest routine use of transbronchial biopsy with or without BAL (17). Should this diagnostic modality fail, along with inconclusive high-resolution CT, the standard practice is progression to lung biopsy.
Given the patient population at the NIH, infections may not be detected based solely on clinical symptoms but rather by imaging evidence in the more severe stages demanding prompt diagnosis for appropriate treatment.

Much like the case with any other clinical tool, there is no clear agreement on the appropriate use of the BAL (18). Because the BAL procedure is associated with low morbidity and may promise a yield of clinical importance, physicians may perform a lavage under a variety of indications. The clearest indication for using BAL is in the diagnosis of opportunistic infections in the immunocompro-

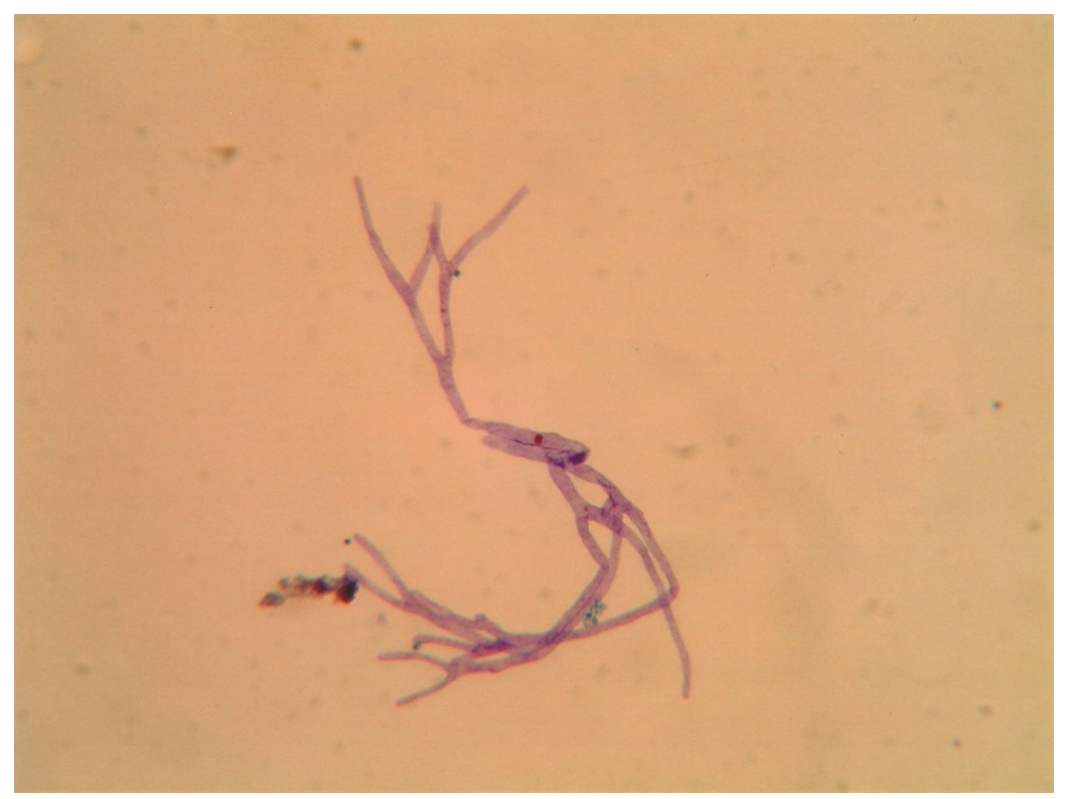

FIGURE 5. LFNA: delicate fungal forms with dichotomous branching. Aspergillus (1000×, Diff-Quik staining). 
TABLE 2. $\chi^{2}$ Test for Independence. Count of Episodes

\begin{tabular}{lccc}
\hline \multicolumn{1}{c}{ Result } & \multicolumn{2}{c}{ Procedure } & \multirow{2}{*}{ Total } \\
\cline { 2 - 3 } & BAL & LFNA & \\
\hline Actual data & & & \\
$\quad$ No infection or malignancy found & 51 & 34 & 85 \\
$\quad$ Infection found & 1 & 12 & 13 \\
Malignancy found & 0 & 6 & 6 \\
$\quad$ Total & 52 & 52 & 104 \\
Expected data & 42.5 & 42.5 & - \\
$\quad$ No infection or malignancy found & 6.5 & 6.5 & - \\
$\quad$ Infection found & 3 & 3 & - \\
$\quad$ Malignancy found & & &
\end{tabular}

$\mathrm{p}<0.0001$ with two degrees of freedom, BAL versus LFNA.

BAL, brochoalveolar lavage; LFNA, lung fine needle aspiration.

mised host $(10,13,18)$. BAL is a proven modality in the evaluation of interstitial lung disease (19). Although not as widely accepted in the past, other investigators have deemed BAL to be an important tool for patients in whom malignancy of the lung is suspected (20). The diagnostic yield in past studies for BAL has ranged from 65 to $76 \%$ (21-23) for confirming patients having carcinoma of the lung. In our study, a variety of indications were given on request for evaluation of cytologic specimens. Specifically, a consolidation or infiltrate was the indication in $60 \%$ of the cases, whereas a mass or cavitary lesion was the indication for $29 \%$ of the cases.

Although a majority of the specimens were evaluated for interstitial lesions, a sizable group of cases was evaluated for localized lesions. Regardless of the indication, LFNA at our institution prevailed in identifying the infectious agent or malignancy, at least in a third of the cases, whereas the BAL was negative. Two factors may have played some part in the lower diagnostic yield of the BAL procedures in our study. The first factor relates to the volume of BAL fluid submitted to the cytopathology lab, whereas the second factor relates to the vasotropism of the Aspergillus species.

The BAL technique, including total volume of saline instilled, varies widely among investigators and clinicians (24). Generally, 200 to $300 \mathrm{~mL}$ of saline is used to sample the subsegmental areas of the lung under investigation (18). The highest volume received on any case during this study was $50 \mathrm{~mL}$ and the lowest volume received was $3 \mathrm{~mL}$, whereas the median volume was $12 \mathrm{~mL}$. These volumes are low compared with the above-stated optimal volume, considering a portion of the sample is divided by some fraction to allow both the microbiology and the cytology departments to receive an amount adequate for processing and analysis. Given the poor condition of many of the patients admitted to the NIH clinical center, it is possible that many of the patients could not tolerate a lavage during these BAL procedures that would yield an optimal amount of fluid for investigation. Perhaps these generally low volumes in turn lead to decreased diagnostic yields for the BAL procedures.
The second reason for the lower diagnostic yield of a BAL relates to the pathophysiology of the Aspergillus species. It has been reported that the results of a BAL are positive in $80 \%$ of patients who have CT findings consistent with airway-invasive aspergillosis compared with a much lower yield of only $18 \%$ of patients who have CT findings consistent with angioinvasive aspergillosis (25). Airway-invasive aspergillosis is characterized by the presence of bronchitis, bronchiolitis, and bronchopneumonia (26); thus, such an infection may likely yield a diagnostic BAL. On the other hand, angioinvasive aspergillosis is characterized by the presence of hemorrhagic infarcts and large nodules and is less likely to demonstrate a positive BAL (25). The CT findings of angioinvasive aspergillosis show nodules, frequently with a halo of ground-glass attenuation, mass-like infiltrates, and areas of segmental consolidation caused by hemorrhagic infarcts (27-29). This differs from the nonspecific CT findings of airway-invasive aspergillosis, usually showing the presence of bronchiolitis and peribronchial consolidation due to bronchopneumonia (30).

This angioinvasive versus airway-invasive behavior of the aspergillus fungus may have had some bearing on our study. Twelve of the 14 infectious agents identified in this series were aspergillus. Comprehensive comparisons of NIH radiological studies to the particular cases cannot be made because all patients did not receive uniform CT or roentgenogram workups. However, given the difference in yield between BAL and LFNA in this current study, it is highly likely that some of the cases of aspergillosis had only an angioinvasive infection, allowing a negative BAL but a positive LFNA.

Assuming that the typical protocol is followed, a patient subject to LFNA has probably already undergone BAL. Therefore, collecting data for analysis on a patient having closely timed BAL and LFNA might represent a selection bias against BAL because progression to LFNA would not occur should the BAL confirm infection or neoplasia. In our study, we identified 22 episodes (of 52 total) in which the LFNA was performed either on the same day as or before the BAL. In 6/22 of those episodes, either an infectious agent or a neoplasia was identified. Thus, for at least a portion of patient episodes in our database, a selection bias against BAL was less likely with the LFNA taking place before or on the same day as the BAL.

As described previously, cytology is useful for the identification of infectious organisms in the pulmonary samples from patients with severe immunodeficiency syndromes (16). The data from this study support previous findings that identification of fungus by LFNA is superior to that of BAL (15). In addition to infectious agents, this study identified neoplasia using LFNA with greater success than with BAL. Although the patient population, again, 
may play a role in the findings, the results may also be highly dependent on the location of the lesion and the operator dependency of the interventional radiologist.

Due to limited access to the medical records, we could not enrich our database with facts related to cost comparisons for the procedures and side effects or reactions by the patients to the procedures. Additionally, we could document only the interval between the BAL and LFNA procedures. Unfortunately, we were not able to discover the clinical reasons that one procedure followed another, such as expectations of different findings between modalities and urgency of the need for a diagnosis.

Although the results of this study may be interesting, the findings must be tempered with the fact that a disease process suspected clinically will largely drive the modality used to assess respiratory cytology. Our findings apply to an immunocompromised population, but we do not believe that such findings can be broadly generalized to all other patient groups. We acknowledge that the underlying disease process and clinical context should be the force motivating the respiratory workup and that not all workups should automatically begin with LFNA and/or BAL. However, there are appropriate situations, such as discrete pulmonary lesions and suspected metastatic lesions to the lung, in which LFNA would be the preferred modality for obtaining cytology specimens. We conclude, based on data from our patient population, that LFNA is the superior method for the cytologic diagnosis of pulmonary pathology amenable to cytologic examination.

\section{REFERENCES}

1. Torres A, el-Eliary M. Invasive diagnostic techniques for pneumonia: protected specimen brush, bronchoalveolar lavage, and lung biopsy methods. Infect Dis Clin North Am 1998;12:703-22.

2. Djukanovic R, Dahl R, Jarjour N, Aalbers R. Safety of biopsies and bronchoalveolar lavage. Eur Respir J Suppl 1998;26:39S-41S.

3. vanSonnenberg E, Lin AS, Deutsch AL, Mattrey RF. Percutaneous biopsy of difficult mediastinal, hilar, and pulmonary lesions by computed tomographic guidance and modified coaxial technique. Radiology 1983;148:300-2.

4. Herman PG, Hessel SJ. The diagnostic accuracy and complications of closed lung biopsies. Radiology 1977;125:11-4.

5. Orell SR, Sterrett GF, Walters MN-I, Whitaker D. Manual and atlas of fine needle aspiration cytology. 2nd ed. Edinburgh, Scotland: Churchill Livingstone; 1992.

6. Sterrett GF, Whitaker D, Glancy J. Fine needle aspiration of lung, mediastinum, and chest wall. Pathol Annu 1982;17 Pt 2:197-228.

7. Berquist TH, Bailey PB, Cortese DA, Miller WE. Transthoracic needle biopsy. Accuracy and complications in relation to location and type of lesion. Mayo Clin Proc 1980;55:475-81.

8. Fraire AE, Underwood RD, McLarty JW, Greeberg SD. Conventional respiratory cytology versus fine needle aspiration cytology in the diagnosis of lung cancer. Acta Cytol 1991;35:385-8.
9. Steffee CH, Segletes LA, Geisinger KR. Changing cytologic and histologic utilization patterns in the diagnosis of 515 primary lung malignancies. Cancer 1997;81:105-15.

10. DeFine LA, Saleba KP, Gibson BB, Wesseler TA, Baughman R. Cytologic evaluation of bronchoalveolar lavage specimens in immunosuppressed patients with suspected opportunistic infections. Acta Cytol 1987;31:235-42.

11. Prior C, Klima G, Gattreinger C, Aigner F, Margreiter R. Cell profiles in serial bronchoalveolar lavage after human heartlung transplantation. Acta Cytol 1992;36:19-25.

12. Stover DE, Zaman MB, Hajdu SI, Lange M, Gold J, Armstrong D. Bronchoalveolar lavage in the diagnosis of diffuse pulmonary infiltrates in the immunosuppressed host. Annu Intern Med 1984;101:1-7.

13. Stover DE, White DA, Romano PA, Gellene RA. Diagnosis of pulmonary disease in acquired immune deficiency syndrome (AIDS). Role of bronchoscopy and bronchoalveolar lavage. Am Rev Respir Dis 1984;130:659-62.

14. Meischl C, Roos D. The molecular basis of chronic granulomatous disease. Springer Semin Immunopathol 1998;19:417-34.

15. Vezza PR, Wilder AM, Holland SM, Abati A. Detection of fungal organisms in pulmonary cytology samples of chronic granulomatous disease: a comparison of alternative techniques. Diagn Cytopathol 2001;24:226-7.

16. Abati A, Cajigas A, Holland SM, Solomon D. Chronic granulomatous disease of childhood: respiratory cytology. Diagn Cytopathol 1996;15:98-102.

17. Cushley M, Davidson A, du Bois R, Flower C, Greening A, Ibrahim N, et al. Diagnosis and assessment of diffuse parenchymal lung disease. Thorax 1999;54(Suppl 1):S1-14.

18. Linder J, Rennard SI. Bronchoalveolar lavage. Chicago, IL: American Society of Clinical Pathologists; 1988.

19. Daniele RP, Elias JA, Epstein PE, Rossman MD. Bronchoalveolar lavage: role in the pathogenesis, diagnosis, and management of interstitial lung disease. Ann Intern Med 1985;102:93-108.

20. Rennard SI. Bronchoalveolar lavage in the assessment of primary and metastatic lung cancer. Respiration 1992; 59(Suppl 1):41-3.

21. Semenzato G, Poletti V. Bronchoalveolar lavage in lung cancer. Respiration 1992;59(Suppl 1):44-6.

22. Pirozunski M. Bronchoalveolar lavage in the diagnosis of peripheral, primary lung cancer. Chest 1992;102:372-4.

23. Linder J, Radio SJ, Robbins RA, Ghafouri M, Rennard SI. Bronchoalveolar lavage in the cytologic diagnosis of carcinoma of the lung. Acta Cytol 1987;31:796-801.

24. Crystal RG, Reynolds HY, Kalica AR. Bronchoalveolar lavage. The report of an international conference. Chest 1986;90:122-31.

25. Brown MJ, Worthy SA, Flint JDA, Müller NL. Invasive aspergillosis in the immunocompromised host: utility of computed tomography and bronchoalveolar lavage. Clin Radiol 1998;53:255-7.

26. Okudaira M, Schwarz J. Tracheobronchopulmonary mycoses caused by opportunistic fungi, with particular reference to aspergillosis. Lab Invest 1962;11:1053-64.

27. Hruban RH, Meziane MA, Zerhouni EA, Wheeler PS, Dumler JS, Hutchins GM. Radiologic-pathologic correlation of the CT halo sign in invasive pulmonary aspergillosis. J Comput Assist Tomogr 1987;11:534-6.

28. Gefter WB. The spectrum of pulmonary aspergillosis. J Thorac Imaging 1992;7:56-74.

29. Kuhlman JE, Fishman EK, Burch PA, Karp JE, Zerhouni EA, Siegelman SS. CT of invasive pulmonary aspergillosis. AJR Am J Roentgenol 1988;150:1015-20.

30. Logan PM, Primack SL, Miller RR, Muller NL. Invasive aspergillosis of the airways: radiographic, CT, and pathologic findings. Radiology 1994;193:383-8. 